\title{
MicroRNA-183 suppresses the vitality, invasion and migration of human osteosarcoma cells by targeting metastasis-associated protein 1
}

\author{
XIAOYA SUN ${ }^{1}$, YAN XU $^{1}$, SHANFENG ZHANG ${ }^{1}$, XINJIE LI $^{1}$, YADONG WANG ${ }^{1}$, \\ YAN ZHANG $^{2}$, XUEFENG ZHAO ${ }^{1}$, YUEBAI LI ${ }^{1}$ and YISHENG WANG ${ }^{2}$ \\ ${ }^{1}$ Department of Biochemistry and Molecular Biology, School of Basic Medical Sciences, \\ Zhengzhou University, Zhengzhou, Henan 450001; ${ }^{2}$ Department of Orthopedic Surgery, \\ The First Affiliated Hospital of Zhengzhou University, Zhengzhou, Henan 450052, P.R. China
}

Received October 22, 2017; Accepted March 1, 2018

DOI: $10.3892 /$ etm.2018.6068

\begin{abstract}
The aim of the present study was to investigate the effects of microRNA (miR)-183 on vitality, invasion, metastasis and apoptosis in osteosarcoma (OS) cells, mediated by its binding to metastasis-associated protein 1 (MTA1). A dual luciferase reporter assay was performed to determine whether MTA1 was a direct target of miR-183. Cell Counting Kit-8, Transwell, scratch-wound healing, fluorescence-activated cell sorting andterminal deoxynucleotidyl transferase dUTP nick end labeling assays were also performed to investigate the effects of miR-183 expression on the proliferation, invasion, migration and apoptosis of MG63 cells. It was demonstrated that that MTA1 expression levels were significantly higher in OS tissues and MG63 cells compared with corresponding adjacent noncancerous tissues and normal cells, respectively, while miR-183 expression levels were significantly lower (both $\mathrm{P}<0.05)$. Furthermore, miR-183 overexpression downregulated MTA1 levels and inhibited cell proliferation $(\mathrm{P}<0.05)$, migration $(\mathrm{P}<0.05)$ and invasion $(\mathrm{P}<0.01)$, as well as promoting apoptosis $(\mathrm{P}<0.01)$ by binding to the 3 '-untranslated region of MTA1. These results indicate that miR-183 inhibits the vitality, invasion, migration and apoptosis of the OS cell line MG63 by targeting MTA1. These findings may contribute to the development of novel clinical therapeutic approaches for the treatment of OS.
\end{abstract}

\section{Introduction}

Osteosarcoma (OS) is a bone tumor that typically affects children and adolescents $<20$ years old. OS is characterized by

Correspondence to: Professor Yuebai Li, Department of Biochemistry and Molecular Biology, School of Basic Medical Sciences, Zhengzhou University, 100 Kexue Road, Zhengzhou, Henan 450001, P.R. China

E-mail: liyuebai@zzu.edu.cn

Key words: osteosarcoma, microRNA-183, metastasis-associated protein 1 , metastasis, invasion, apoptosis lung metastasis and poor prognosis, posing a serious threat to health (1). In recent years, research into the molecular changes associated with cancer has received increasing attention (2). The identification of valuable novel biomarkers and potential molecular-level treatment approaches for OS are expected to improve future diagnosis and treatment (3).

Metastasis-associated protein 1 (MTA1) is a chromatin remodeling factor that forms part of the nucleosome remodeling and histone deacetylase (NuRD) complex, along with MTA2 and MTA3 and is crucial for gene expression, cell survival and promoting the hypoxic response as an oncogene upregulated in human cancer (4). A previous study demonstrated that MTA1 promotes non-small-cell lung cancer cell epithelial-mesenchymal transition and metastasis (5). Kim and Park (6) also reported the high-level expression of MTA1 in OS.

MicroRNAs (miRNA/miRs) are a group of endogenous small (19-25-nucleotide) non-coding RNAs. Differential expression of miRNAs has been described in almost every type of tumor, and so miRNAs have potential as diagnostic or prognostic markers $(7,8)$. In the present study, it was observed that miR-183 functioned as a tumor suppressorand served a significant role in OS cell proliferation, migration and apoptosis by binding to a target gene. MiRNAs post-transcriptionally silence complementary target genes and increasing evidence suggests that miR-183 functions as a tumor suppressor (9). For example, miR-183 has been reported to function as an oncogene to suppress neuropathic pain and the expression of the $\alpha$-amino-3-hydroxy-5-methyl-4-isoxazolepropionic acid receptor (10).

The aim of the present study was to investigate the effects of miR-183 on cellular processes, including metastasis, invasion and apoptosis and to evaluate the mechanisms by which miR-183 regulates its direct target MTA1 in MG63 cells.

\section{Materials and methods}

Tumor sample collection. Paired OS tumor tissues and adjacent normal tissues were obtained from patients $(n=25)$, including 14 male and 11 female (mean age 16 years; range, 6-58 years) who were treated at the First Affiliated Hospital of Zhengzhou 
University (Henan, China) between September 2014 and June 2016, in accordance with an institutionally approved protocol. All samples were obtained from primary OS patients who were not subjected to chemotherapy or radiotherapy and were not suffering from any other diseases. All patients were diagnosed with OS on the basis of clinicopathological findings. All tissues were flash frozen in liquid nitrogen at the time of surgery and stored at $-80^{\circ} \mathrm{C}$ until use. All procedures were conducted in accordance with the principles of the Declaration of Helsinki. Informed consent was obtained from patients themselves or their families and the procedures were approved by the ethics committee of Zhengzhou University.

Cell lines and cell culture. The normal human osteoblastic cell line hFOB 1.19 and the human OS cell line MG63 were purchased from the Cell Culture Center of the Chinese Academy of Medical Sciences (Beijing, China). MG63 cells were maintained in RPMI 1640 medium (Gibco; Thermo Fisher Scientific, Inc., Waltham, MA, USA) containing 10\% fetal bovine serum (FBS; Sigma-Aldrich, USA), $100 \mathrm{U} / \mathrm{ml}$ penicillin and $100 \mu \mathrm{g} / \mathrm{ml}$ streptomycin at $37^{\circ} \mathrm{C}$ in a humidified incubator containing $5 \% \mathrm{CO}_{2}$. hFOB 1.19 cells were grown in Dulbecco's modified Eagle's medium/F12 (1:1; Gibco; Thermo Fisher Scientific, Inc.) supplemented with $10 \%$ FBS, $100 \mathrm{U} / \mathrm{ml}$ penicillin and $100 \mu \mathrm{g} / \mathrm{ml}$ streptomycin at $37^{\circ} \mathrm{C}$ in a humidified incubator containing $5 \% \mathrm{CO}_{2}$.

RNA extraction and reverse transcription-quantitative polymerase chain reaction ( $R T-q P C R)$. Total RNA was purified from tissue samples and cultured cells using TRIzol reagent (Invitrogen; Thermo Fisher Scientific, Inc.) according to the manufacturer's protocol. RT was performed at $42^{\circ} \mathrm{C}$ for $2 \mathrm{~min}$, $37^{\circ} \mathrm{C}$ for $15 \mathrm{~min}$ and $85^{\circ} \mathrm{C}$ for $5 \mathrm{sec}$ using a Takara RNA PCR kit (Takara Biotechnology Co., Ltd., Dalian, China) according to the manufacturer's protocol. qPCR was performed using SYBR Premix Ex TaqII (Takara Biotechnology Co., Ltd.) and an Applied Biosystems 7500 Real-time PCR System (Thermo Fisher Scientific, Inc.) according to the manufacturer's protocol. All reactions were performed in triplicate and thermocycling conditions were as follows: $95^{\circ} \mathrm{C}$ for $30 \mathrm{sec}$ followed by 40 cycles of $95^{\circ} \mathrm{C}$ for $5 \mathrm{sec}$ and $60^{\circ} \mathrm{C}$ for $30 \mathrm{sec}$, with a final dissociation stage for $1 \mathrm{~h}$ at $4^{\circ} \mathrm{C}$. U6 small nuclear RNA and $\beta$-actin mRNA were used as internal controls. The relative level of miRNA expression and mRNA expression was calculated using the $2^{-\Delta \Delta \mathrm{Cq}}$ method (11). The primers used were designed and synthesized by Sangon Biotech Co., Ltd. (Shanghai, China) and are listed in Table I.

miRNA transfection. ThemiR-183 mimic and negative control miRNA used in the current study were obtained from Shanghai GenePharma Co., Ltd. (Shanghai, China). MG63 cells were seeded in 24 -well plates $\left(2 \times 10^{5}\right.$ cells/well) and grown to $\sim 80 \%$ confluence as described above. Cells were transiently transfected using Lipofectamine ${ }^{\circledR} 2000$ (Invitrogen; Thermo Fisher Scientific, Inc.), according to the manufacturer's protocol. The following groups of cells were prepared: Cells treated with vehicle only (control group); cells transfected with a scramble miR-183 sequence [negative control (NC) group]; and cells transfected with miR-183 mimic (miR-183 group). The transfection efficiency of miR-183 mimic was tested using
RT-qPCR as described above, after 1-2 days and cells were used for further experiments.

Cell Counting Kit-8 (CCK-8) cell viability assay. Transfected MG63 cells were seeded in 96 -well plates $\left(5 \times 10^{3}\right.$ cells/well, five replicate wells per group), cultured for 1-2 days and subsequently subjected to a cell viability assay using a CCK-8 (Dojindo Molecular Technologies, Inc., Kumamoto, Japan) to detect viable cells on days 1-5. In brief, cells were incubated with $10 \mu \mathrm{l} \mathrm{CCK}-8$ solution for $48 \mathrm{~h}$ at $37^{\circ} \mathrm{C}$ in a humidified atmosphere containing $5 \% \mathrm{CO}_{2}$ until a visible color conversion occurred. Absorbance values were measured at $490 \mathrm{~nm}$ and proliferation curves were plotted.

Transwell invasion assay. Following $48 \mathrm{~h}$ of culture, transfected cells were collected and resuspended in RPMI 1640 medium at $2 \times 10^{5}$ cells $/ \mathrm{ml}$. The cell suspension $(200 \mu \mathrm{l})$ was transferred to the upper chamber of a Transwell Permeable Support device (Costar; Corning Incorporated, Corning, NY, USA) with a membrane insert containing $8-\mu \mathrm{m}$ pores and incubated at $37^{\circ} \mathrm{C}$ in a humidified incubator containing 5\% $\mathrm{CO}_{2}$. RPMI 1640 culture medium $(500 \mu \mathrm{l})$ containing $10 \%$ FBS was added to the lower chamber of the Transwell device. Following 24 or $48 \mathrm{~h}$ of incubation, cells on the upper surface of the membrane were removed using a cotton swab. The cells that had migrated to the lower surface of the membrane were fixed with $4 \%$ paraformaldehyde for $15 \mathrm{~min}$ at room temperature, washed three times with PBS and stained with hematoxylin for $15 \mathrm{~min}$ at room temperature. Cells were subsequently mounted and air-dried and the number of cells that had invaded the lower surface of the membrane was counted in three randomly selected fields under a light microscopic at x200 magnification.

Scratch-wound healing assay. At $24 \mathrm{~h}$ following transfection, cells were seeded in 6 -well plates $\left(2 \times 10^{3}\right.$ cells/well) and allowed to grow overnight to $80-90 \%$ confluence at $37^{\circ} \mathrm{C}$. The cell layer was then scratched using a sterile $10-\mu 1$ pipette tip. The wounded monolayer was washed twice with PBS and fresh serum-free culture medium was added. At 24 and $36 \mathrm{~h}$ after wounding, micrographs were captured with a light microscope (original magnification, x100). All experiments were repeated in triplicate.

Apoptosis assay. Fluorescence-activated cell sorting assays were conducted to assess apoptosis. At $36 \mathrm{~h}$ following transfection, cells were harvested and diluted in $500 \mu \mathrm{l}$ PBS (1x10 ${ }^{6}$ cells $/ \mathrm{ml}$; Shanghai BestBio Biotechnology, Shanghai, China) and subsequently stained with Annexin V-fluorescein isothiocyanate and propidium iodide (Beyotime Institute of Biosciences, Haimen, China) at room temperature in the dark for $15 \mathrm{~min}$. A calibrated FACSCalibur flow cytometer (BD Biosciences, Franklin Lakes, NJ, USA) equipped with Cell Quest software 5.1 (BD Biosciences) was used to analyze the cells.

To further analyze apoptosis, a TUNEL assay was performed. Following $36 \mathrm{~h}$ of culture in a 6-well plate (as above), transfected cells were subjected to a Dead End ${ }^{\mathrm{TM}}$ Fluorometric TUNEL System (Promega Corp., Madison, WI, USA) assay in accordance with the manufacturer's protocol to detect apoptotic cells. Briefly, cells were washed twice with 
Table I. Primers used for reverse transcription-quantitative polymerase chain reaction.

\begin{tabular}{lll}
\hline Gene & Direction & Sequence $\left(5^{\prime}-3^{\prime}\right)$ \\
\hline U6 & Forward & TCCGATCGTGAAGCGTTC \\
& Reverse & GTGCAGGGTCCGAGGT \\
Human microRNA-183 & Forward & ACATCTCCAGCACCCTCATC \\
& Reverse & TAGATGGGCACAGTGTGGGTG \\
Metastasis-associated protein 1 & Forward & CTGCGAGTATGGCACTGGTAGAA \\
& Reverse & ATCCAGTGCAGGGTCCGAGG \\
& Forward & CGGATTCTCCATTTCCTCTTC \\
& Reverse & ACATCTCCAGCACCCTCATC
\end{tabular}

A

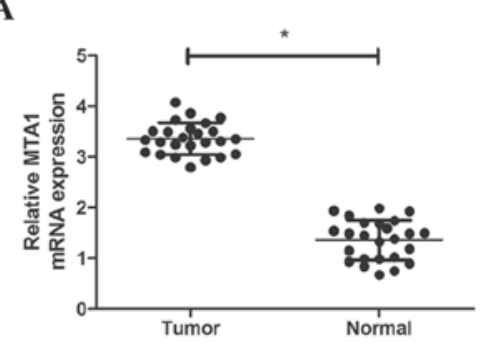

D

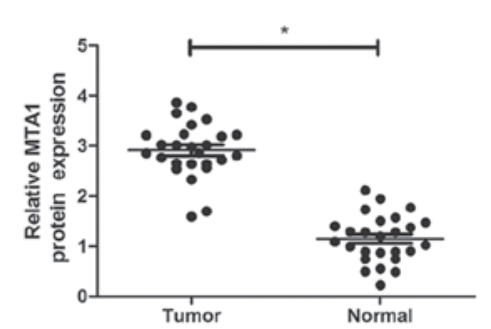

B

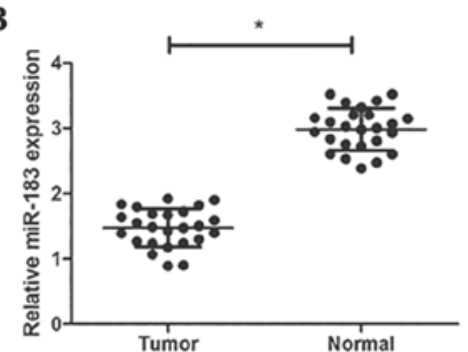

E

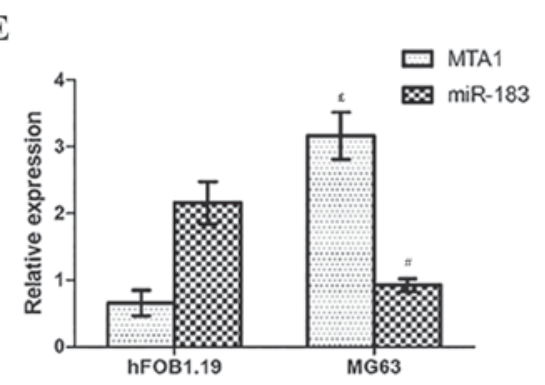

C

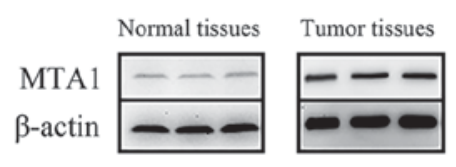

F

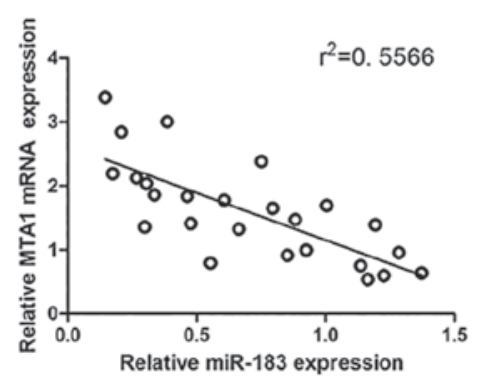

Figure 1. RT-qPCR was used to assess the relative levels of (A) MTA1 mRNA and (B) miR-183 in OS tissues compared with matched normal paracancerous tissues. (C) Western blotting was used to detect MTA1 protein levels in OS tissues and matched normal paracancerous tissues and (D) quantified. (E) RT-qPCR was used to assess the relative levels of MTA1 mRNA and miR-183 in the OS cell line MG63 compared with the normal human cell line hFOB 1.19. (F) Correlation analysis was performed to assess the association between miR-183 and MTA1 expression levels. * $\mathrm{P}<0.05$. ${ }^{\mathrm{f}} \mathrm{P}<0.05 \mathrm{vs}$. the MTA1 hFOB1.19 group. ${ }^{*} \mathrm{P}<0.05$ vs. the miR-183 hFOB1.19 group. RT-qPCR, reverse transcription-quantitative polymerase chain reaction; miR, microRNA; MTA1, metastatic tumor protein 1; OS, osteosarcoma.

PBS, fixed with $4 \%$ paraformaldehyde for $20 \mathrm{~min}$ at room temperature and treated with $50 \mu 1$ of the TUNEL reaction solution, $50 \mu \mathrm{l}$ of the enzyme solution and $450 \mu \mathrm{l}$ of the labeling solution at $37^{\circ} \mathrm{C}$ in the dark for $1 \mathrm{~h}$. The cells were then observed by fluorescence microscopy at magnification, x200 (Olympus Corporation, Tokyo, Japan).

Bioinformatics analysis. Using starBase V2.0 (http://starbase. sysu.edu.cn/), TargetScan 7.1 (http://www.targetscan.org/) and miRanda(http://www.microrna.org)bioinformatics software, the target genes were identified. The results obtained from the software packages were considered and the 3'-untranslated region (3'-UTR) region of MTA1 mRNA was selected as the sequence most likely to be complementary to miR-183.

Dual luciferase reporter assay. PCR was used to amplify the region of the MTA1 gene that included putative binding sites for miR-183 from MG63 cells with a Taq DNA Mix kit (Takara Biotechnology Co., Ltd.). Overlap-extension PCR was used to amplify the mutant segment of the MTA1 mRNA 3'UTR. The primers usedwere as follows: Wild type forward 5'TTT CTCGAGGCCGAGGAGTTGTCGTTTTTAGCTT3', and wild type reverse, 5'TTCTCTAGACTTGAAAAGACAAGG GCCAACCCCG3'; mutantforward, 5'TTGAAGCACGGT ATTTAAATTTTATTTTTATTACTTTTTTTGTAG3' and mutantreverse, 5'AATACCGTGCTTCAACAGAACACA ACAAAACCTTAGGGCCCGGCC3'. The PCR steps were as follows: Initial denaturation for $3 \mathrm{~min}$ at $95^{\circ} \mathrm{C}$; 34 cycles of denaturation for $35 \mathrm{sec}$ at $95^{\circ} \mathrm{C}$, annealing for $35 \mathrm{sec}$ at $55^{\circ} \mathrm{C}$ and elongation for $60 \mathrm{sec}$ at $72^{\circ} \mathrm{C}$; and a final extension step for $60 \mathrm{~min}$ at $10^{\circ} \mathrm{C}$. Amplified fragments were inserted into a pmir-GLO plasmid (Promega Corp.) downstream of the luciferase gene to create the recombinant vectors pGLO-MAT1-wt (wild-type reporter vector) and pGLO-MTA1-mut (mutant 
A

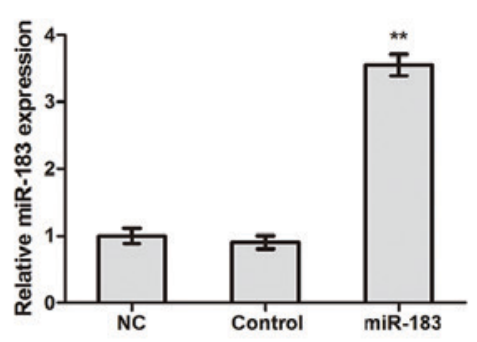

D

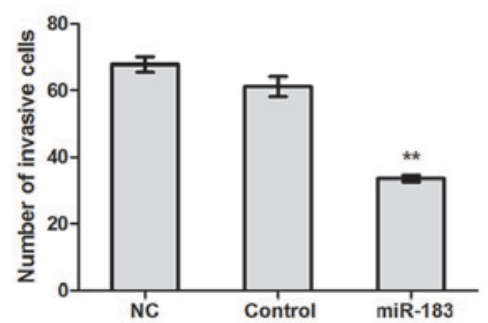

B

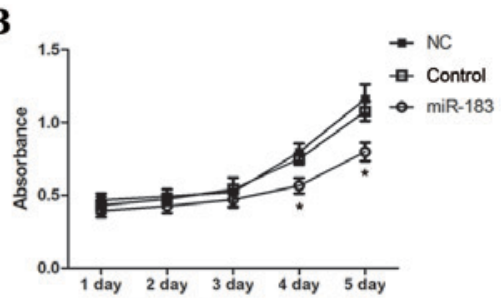

$\mathbf{E}$

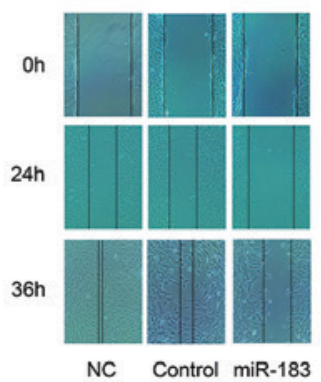

C

$24 \mathrm{~h}$
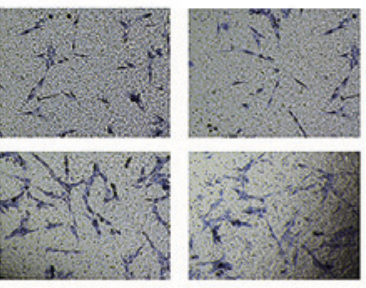

Control
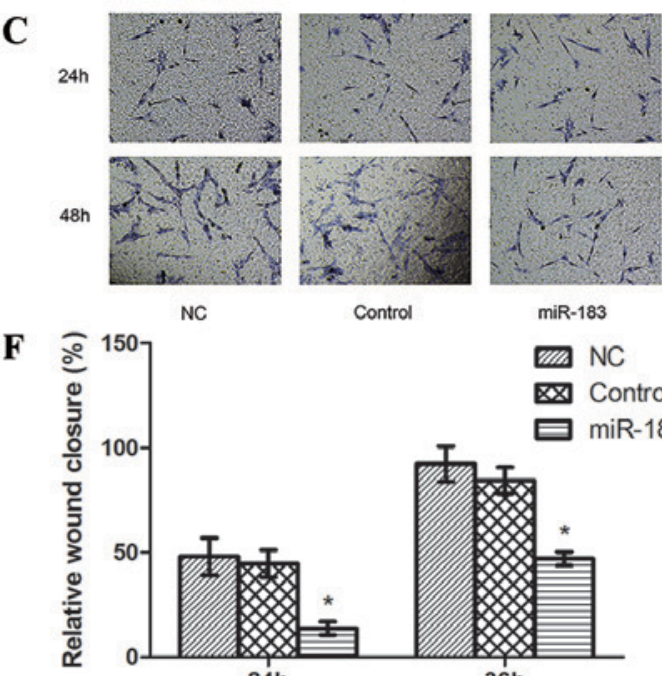

NC

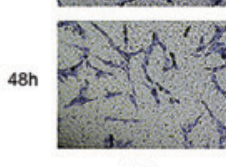

$24 \mathrm{~h}$

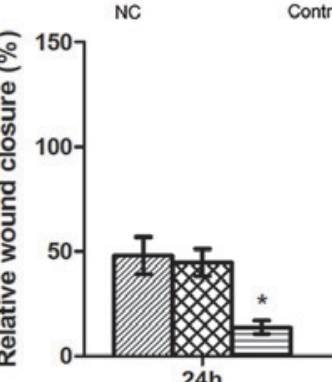

miR-183

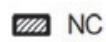

四 Control 曰 miR-183

Figure 2. miR-183 upregulation inhibits proliferation, invasion and migration in osteosarcoma cells in vitro. (A) The transfection efficiency of miR-183 mimic was assessed using reverse transcription-quantitative polymerase chain reaction. (B) A Cell Counting Kit- 8 assay was used to assess proliferation in miR-183 mimic-transfected, control and NC cells. (C) The effect of the miR-183 mimic on cell invasion was detected using a Transwell assay (magnification x200) and (D) quantified. A scratch-wound healing assay (magnification, $\mathrm{x} 100)$ was performed, (E) assessed at 0,24 and $36 \mathrm{~h}$ and $(\mathrm{F})$ quantified. ${ }^{*} \mathrm{P}<0.05$ and ${ }^{* *} \mathrm{P}<0.01$. miR, microRNA; NC, negative control.

reporter vector). MG63 cells were cultured for $48 \mathrm{~h}$ in 96-well plates $\left(2 \times 10^{5}\right.$ cells $\left./ \mathrm{ml}\right)$ and the miRNA and reporter plasmid were cotransfected into the cells using Lipofectamine ${ }^{\circledR} 2000$ reagent according to the manufacturer's protocol. At $24 \mathrm{~h}$ following transfection, luciferase activity was determined using a Dual Luciferase Reporter Assay System (Promega Corp.) according to the manufacturer's protocol.

Western blotting. Western blotting was performed to examine MTA1 protein expression levels in the OS tissue samples and MG63 cells. Cells were incubated and lysed in radioimmunoprecipitation assay buffer containing phenylmethanesulfonyl fluoride (Beyotime Institute of Biotechnology) to extract the total protein, following which the total protein concentration was quantified using a Bicinchoninic Acid Protein Assay kit (Beyotime Institute of Biotechnology). The procedure for western blotting was as follows: Extracted proteins (10 $\mu \mathrm{l}$ per lane)were separated by $10 \%$ SDS-PAGE and transferred to polyvinylidene difluoride membranes. The membranes were subsequently blocked with 5\% non-fat milk at room temperature for $2 \mathrm{~h}$, rinsed four times with Tris-buffered saline containing Tween-20 (TBST) and incubated with primary antibodies against MTA1 (cat. no. sc-373765; diluted 1:1,000) and $\beta$-actin (cat. no. sc-130065; diluted 1:3,000) (both Santa Cruz Biotechnology, Inc., Dallas, TX, USA) overnight at $4^{\circ} \mathrm{C}$. Membranes were then washed in TBST four times and incubated with horseradish peroxidase-conjugatedgoat anti-mouse secondary immunoglobulin G (cat. no. sc-2005; diluted 1:2,500; Santa Cruz Biotechnology, Inc.) for $2 \mathrm{~h}$ at room temperature. The membranes were then washed four times (5 min per wash) with TBST and signals from immunoreactive bands were quantified with a chemiluminescence detection kit (GE Healthcare Life Sciences, Little Chalfont, UK). AlphaView3.3.0 software (ProteinSimple; Bio-Techne, Minneapolis, MN, USA) was used for densitometry analysis. $\beta$-actin was used as an internal reference to confirm equal protein loading.

Statistical analysis. All statistical analyses including correlation analysis were performed using SPSS 19.0 software (IBM Corp., Armonk, NY, USA) and data are presented as the mean \pm standard deviation. The Student's t-test, Pearson correlation analysis, one-way analysis of variancewith Dunnett's post hoc test were used to evaluate differences between groups. $\mathrm{P}<0.05$ was considered to indicate a statistically significant difference.

\section{Results}

miR-183 and MTA1 expression in human OS tissues and cell lines. RT-qPCR analysis revealed that MTA1 mRNA and MTA1 protein levels were higher in OS tissues compared with normal adjacent tissues, whereas relative miR-183 levels were lower (both $\mathrm{P}<0.05$; Fig. 1A and B). Western blotting revealed that MTA1 was overexpressed in OS tissues compared with the normal paracancerous tissues (Fig. 1C and D). The same patterns were observed in the OS and normal cell lines via the same methods: miR-183 expression was significantly lower in MG63 cells compared with their expression levels in hFOB 1.19 cells, while MTA1 mRNA expression was higher (both $\mathrm{P}<0.05$; Fig. 1E). A negative correlation was also observed between miR-183 and MTA1 expression (Fig. 1F).

miR-183 overexpression impairs proliferation, invasion and migration in MG63 cells in vitro. To determine the potential functional role of miR-183 in OS cell proliferation, migration and invasion, CCK-8, Transwell and scratch-wound healing assays were performed to examine the differences between cells prior to and following transfection with an miR-183 mimic. The results of RT-qPCR revealed that the transfection efficiency of miR-183 mimic-transfected cells was significantly 
A

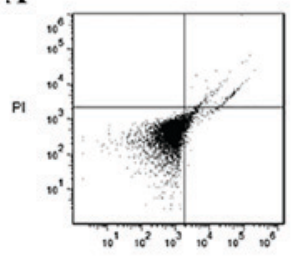

FITC

NC

C

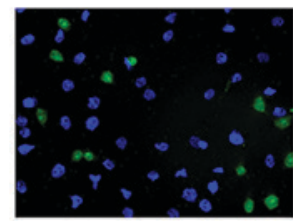

NC

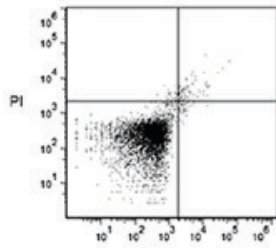

FITC

Control

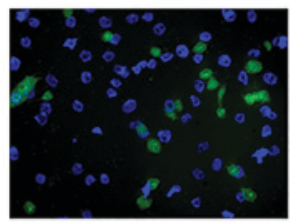

Control

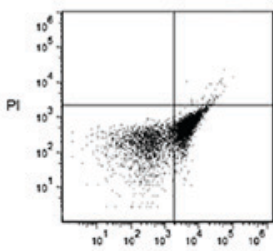

FITC

miR-183

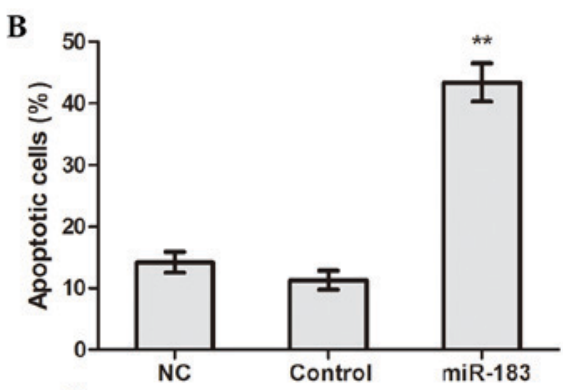

D

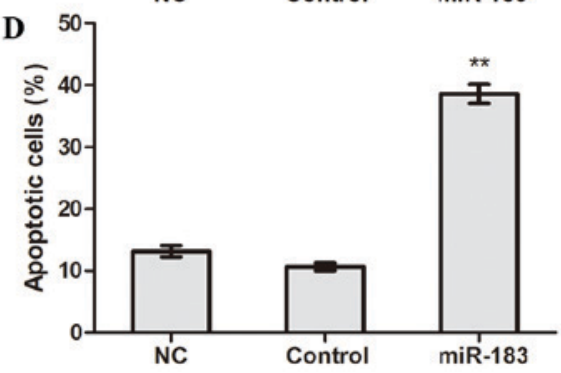

Figure 3. miR-183 promotes apoptosis in osteosarcoma cells. (A) Flow cytometry analysis of transfected cells was performed and (B) quantified. (C) Apoptosis in miR-183 mimic-transfected, control and NC cells was detected using a TUNEL assay (magnification x200) and (D) analyzed. ${ }^{* *} \mathrm{P}<0.01 \mathrm{vs.}$ the control group. miR, microRNA; NC, negative control; PI, propidium iodide; FITC, fluorescein isothiocyanate.

A
5' ... UguguUCUgUUGAAgGUGCCAUU .. 3'
I I I I I I I I I I I I
3' ACUUAAGAUGGU------CACGgUAU 5'
wt-MTA1
5' UgUGUUCUGUUGAAGCACGGAUU 3'
hsa-miR-183
mut-MTA1
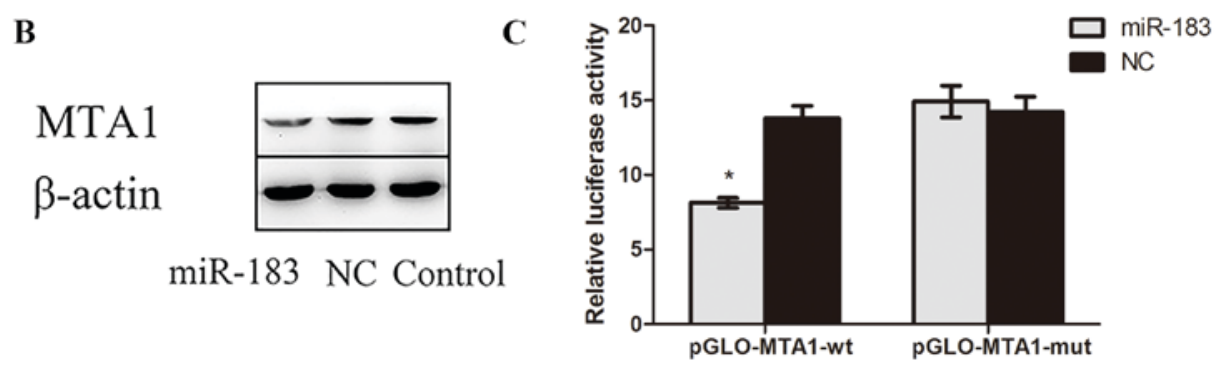

Figure 4. miR-183 binds directly to MTA1. (A) Schematic of predicted miR-183 binding sites in the MTA1 3'-UTR and the mutant sequences of MTA1. The red text indicates the mutant sequence. (B) MTA1 expression in the miR-183 mimic-transfected, NC and control groups was assessed using western blotting with $\beta$-actin as a loading control. (C) A dual luciferase activity was performed to compare osteosarcoma cells transfected with miR-183 mimic and pmirGLO-MTA1 with cells cotransfected with miR-183 negative control and pmirGLO-MTA1. "P<0.05 vs. the miR-183 pGLO-MTA1-mut group. miR, microRNA; MTA1, metastatic tumor protein 1; 3'-UTR, 3'-untranslated region; NC, negative control; wt, wild type; mut, mutant; hsa, human.

higher compared with control and NC cells ( $\mathrm{P}<0.01$; Fig. 2A), whereas no significant differences were observed between the control and NC cells.

The results of a CCK-8 assay revealed a lower absorption value in miR-183 mimic-transfected cells compared with control and NC cells $(\mathrm{P}<0.05$; Fig. $2 \mathrm{~B})$, which suggests that high miR-183 expression may suppress OS cell proliferation. In the Transwell assay, the number of cells that crossed the basement membrane was significantly lower in miR-183 mimic-transfected cells $(33.5924 \pm 0.9857)$ compared with the control $(61.1830 \pm 2.9217)$ and $\mathrm{NC}(67.7391 \pm 2.3318)$ cells $(\mathrm{P}<0.01$; Fig. 2C and D). This suggests that increased miR-183 expression inhibits OS cell invasion. Furthermore, the scratch-wound healing assay revealed that the relative extent of migration of miR-183 mimic-transfected cells was significantly lower compared with the control and $\mathrm{NC}$ cells $(\mathrm{P}<0.05$; Fig. 2E and F), which suggests that miR-183 overexpression impairs OS cell migration.

miR-183 overexpression promotes apoptosis in OS cells in vitro. The apoptosis of transfected cells was detected using flow cytometry. The results revealed that the apoptotic rate of miR-183 mimic-transfected cells was significantly higher compared with control and $\mathrm{NC}$ cells $(\mathrm{P}<0.01$; Fig. $3 \mathrm{~A}$ and $\mathrm{B})$, whereas no significant difference in apoptotic rate was observed between the control and NC cells. TUNEL assay results confirmed the apoptotic rate of miR-183 mimic-transfected cells was higher compared with control and NC cells 
$(\mathrm{P}<0.01$; Fig. 3C and $\mathrm{D})$. These results suggest that elevated miR-183 expression promotes OS cell apoptosis.

miR-183 binds directly to MTA1 and there is a negative association between miR-183 and MTAl expression in OS cells. The complementary sites of MTA1 and miR-183 predicted by bioinformatics software, as well as the mutant sequences of MTA1, are presented in Fig. 4A. The results of western blotting demonstrated that MTA1 protein expression was lower in miR-183 mimic-transfected cells compared with control and NC cells (Fig. 4B), confirming that miR-183 targets MTA1. Furthermore, a dual luciferase reporter assay indicated that luciferase activity in miR-183 mimic and pmirGLO-MTA1-wtcotransfected OS cells was significantly decreased compared with cells cotransfected with scramble miR-183 pmirGLO-MTA1-mut ( $<<0.05$; Fig. 4C). No significant difference in luciferase activity was observed between cells cotransfected with miR-183 negative control and pmirGLO-MTA1-wt and those transfected with miR-183 negative control and pmirGLO-MTA1-mut. Collectively, these results suggest that miR-183 targets the 3'-UTR of MTA1 and negatively regulates its expression.

\section{Discussion}

MTA1 has been established as a DNA damage response protein and has been widely studied in a variety of types of cancer (12). Tuncay Cagatay et al (13) demonstrated that MTA1 enhances zing finger proteins SNAI1 and SNAI2 expression and increases epithelial cadherin expression. Furthermore, they reported that MTA1 overexpression promotes cell proliferation, migration and invasion in colorectal cancer cells (13). In human non-small-cell lung carcinoma and liver cancer cells, MTA1 has been reported to inhibit apoptosis by regulating the NuRD complex, which mediates p53 deacetylation $(14,15)$. Furthermore, a meta-analysis of several tumor types demonstrated that MTA1 may be used as a novel indicator of poor prognosis in patients with tumors $(16,17)$. These findings suggest that MTA1 may be a potential target for cancer therapy. In the present study, it was identified that MTA1 was significantlyupregulated in OS tissues and cells and served as a potential oncogene in OS.

miRNAs silence target genes or induce mRNA degradation by completely or incompletely binding to the 3'-UTR of target mRNAs $(18,19)$. Zhu et al $(20)$ demonstrated that miR-183 downregulates ezrin expression and inhibits the invasion and migration of OS. Additionally, a number of studies have reported that miR-183 inhibits the function of transforming growth factor- $\beta 1$ to induce apoptosis in human hepatoma cells by targeting programmed cell death $4(21,22)$. Other studies have demonstrated that miR-183 levels were abnormally low in a number of cancers, including OS (23-27). In particular, a study by Wang et al (28) identified low expression of miR-183 and high expression of MTA1 in 29 nasopharyngeal carcinoma (NPC) tissues compared with 17 normal nasopharyngeal epithelium tissues; cell experiments using NPC cell lines compared with the human immortalized nasopharyngeal epithelial cell line also obtained the same results. Furthermore, Wang et al (28) reported that miR-183 overexpression inhibits NPC cell proliferation and migration, increases the rate of cellular apoptosis and improves the cytotoxicity induced by the antitumor drug cisplatin by targeting MTA1. These results were verified by a xenograft tumor experiment in vivo (28). To further confirm the reduction in miR-183 expression in OS, miR-183 expression levels in OS and paracancerous tissues were assessed in the present study, as well as the OS cell line MG63 and the normal osteoblast cell line hFOB1.19. The results obtained were in agreement with previously published reports.

To the best of our knowledge, the effects of miR-183 binding to the 3'-UTR of MTA1 have not previously been studied. The results of the present study demonstrate that MTA1 is a direct target gene of miR-183 and that miR-183 negatively regulates the expression of MTA1 by binding to the 3'-UTR. Additionally, low miR-183 expression and high MTA1 expression may serve a role in the pathogenesis of OS. The results of the present study also suggest thatmiR-183 upregulation may reduce MTA1 expression, thereby inhibiting proliferation, invasion and migration as well as promoting apoptosis in OS cells. However, the present study is not without limitations. The central hypothesis was tested in only one cell line and so should be validated in more cell lines as well as xenograft tumor experiments in future studies.

In conclusion, the present study is the first to report that miR-183 regulates MTA1 gene expression in OS. These results may provide a basis for the development of novel treatment targets for the prevention and treatment of OS in the future.

\section{Acknowledgements}

The authors would like to thank Professor Li Yuebai (Zhengzhou University, Zhengzhou, China) for their guidance and support during the experimental project.

\section{Funding}

No fundingwas received.

\section{Availability of data and materials}

All data generated or analyzed during this study are included in this published article.

\section{Authors' contributions}

YL, XS and YX conceived the study, participated in its design and coordination and helped to draft the manuscript. YW, YZ and XL collected the samples. XS, YX, SZ, XL and YW performed some of the experiments and wrote the manuscript. YL, XS, YW and XZ performed the statistical analysis. All authors read and approved the final manuscript.

\section{Ethics approval and consent to participate}

All procedures were conducted in accordance with the principles of the Declaration of Helsinki. Informed consent was obtained from the patients or their families and the procedures were approved by the Ethics Committee of Zhengzhou University. 


\section{Consent for publication}

Informed consent was obtained from all individuals included within the study for the publication of their data.

\section{Competing interests}

The authors declare that they have no competing interests.

\section{References}

1. Garimella R, Tadikonda P, Tawfik O, Gunewardena S, Rowe P and Van Veldhuizen P: Vitamin D impacts the expression of Runx2 Target Genes and Modulates Inflammation, oxidative stress and membrane vesicle biogenesis gene networks in 143B osteosarcoma cells. Int J Mol Sci 18, E642: 2017.

2. Mingardi J, Musazzi L, De Petro G and Barbon A: miRNA editing: New insights into the fast control of gene expression in health and disease. Mol Neurobiol: Feb 19, 2018 (Epub ahead of print).

3. Schadt EE: Molecular networks as sensors and drivers of common human diseases. Nature 461: 218-223, 2009.

4. Kaur E, Gupta S and Dutt S: Clinical implications of MTA proteins in human cancer. Cancer Metastasis Rev 33: 1017-1024, 2014.

5. Li SH, Tian H, Yue WM, Li L, Li WJ, Chen ZT, Hu WS, Zhu YC and Qi L: Overexpression of metastasis-associated protein 1 is significantly correlated with tumor angiogenesis and poor survival in patients with early-stage non-small cell lung cancer. Ann Surg Oncol 18: 2048-2056, 2011.

6. Kim SS and Park YK: Significance of MTA1 in the molecular characterization of osteosarcoma. Cancer Metastasis Rev 33: 981-991, 2014

7. Sadri D, Farhadi S, Shahabi Z and Sarshar S: Expression of vascular endothelial growth factor in odontogenic cysts: Is there any impression on clinical outcome. Open Dent J 10: 752-759, 2016.

8. Matsumoto K, Xavier S, Chen J, Kida Y, Lipphardt M, Ikeda R, Gevertz A, Caviris M, Hatzopoulos AK, Kalajzic I, et al: Instructive role of the microenvironment in preventing renal fibrosis. Stem Cells Translat Med 6: 992-1005, 2017.

9. Fan D, Wang Y, Qi P, Chen Y, Xu P, Yang X, Jin X and Tian X: MicroRNA-183 functions as the tumor suppressor via inhibiting cellular invasion and metastasis by targeting MMP-9 in cervical cancer. Gynecol Oncol 141: 166-174, 2016.

10. Xie X, Ma L, Xi K, Zhang W and Fan D: MicroRNA-183 suppresses neuropathic pain and expression of AMPA receptors by targeting mTOR/VEGF signaling pathway. Cell Physiol Biochem 41: 181-192, 2017.

11. Livak KJ and Schmittgen TD: Analysis of relative gene expression data using real-time quantitative PCR and the 2(-Delta Delta C(T)) method. Methods 25: 402-408, 2001.

12. Li DQ, Pakala SB, Reddy SD, Ohshiro K, Peng SH, Lian Y, Fu SW and Kumar R: Revelation of p53-independent function of MTA1 in DNA damage response via modulation of the p21 WAF1-proliferating cell nuclear antigen pathway. J Biol Chem 285: 10044-10052, 2010.
13. Tuncay Cagatay S, Cimen I, Savas B and Banerjee S: MTA-1 expression is associated with metastasis and epithelial to mesenchymal transition in colorectal cancer cells. Tumour Biol 34: 1189-1204, 2013

14. Moon HE, Cheon H and Lee MS: Metastasis-associated protein 1 inhibits p53-induced apoptosis. Oncol Rep 18: 1311-1314, 2007.

15. Xue Y, Wong J, Moreno GT, Young MK, Côté J and Wang W: NURD, a novel complex with both ATP-dependent chromatin-remodeling and histone deacetylase activities. Mol Cell 2: 851-861, 1998.

16. Oliveto S, Mancino M, Manfrini N and Biffo S: Role of microRNAs in translation regulation and cancer. World J Biol Chem 8: 45-56, 2017.

17. Zhang H, Zhu X, Li N, Li D, Sha Z, Zheng X and Wang H: MiR-125a-3p targets MTA1 to suppress NSCLC cell proliferation, migration, and invasion. Acta Biochim Biophys Sin (Shanghai) 47: 496-503, 2015.

18. Cao JM, Li GZ, Han M, Xu HL and Huang KM: MiR-30c-5p suppresses migration, invasion and epithelial to mesenchymal transition of gastric cancer via targeting MTA1. Biomed Pharmacother 93: 554-560, 2017.

19. Miao Y, Lu M, Yan Q, Li S and Feng Y: Inhibition of proliferation, migration, and invasion by knockdown of pyruvate kinase-M2 (PKM2) in ovarian cancer SKOV3 and OVCAR3 cells. Oncol Res 24: 463-475, 2016.

20. Zhu J, Feng Y, Ke Z, Yang Z, Zhou J, Huang X and Wang L: Down-regulation of miR-183 promotes migration and invasion of osteosarcoma by targeting Ezrin. Am J Pathol 180: 2440-2451, 2012.

21. Gu W, Gao T, Shen J, Sun Y, Zheng X, Wang J, Ma J, Hu XY, $\mathrm{Li} \mathrm{J}$ and Hu MJ: MicroRNA-183 inhibits apoptosis and promotes proliferation and invasion of gastric cancer cells by targeting PDCD4. Int J Clin Exp Med 7: 2519-2529, 2014.

22. Li J, Fu H, Xu C, Tie Y, Xing R, Zhu J, Qin Y, Sun Z and Zheng X: miR-183 inhibits TGF-beta1-induced apoptosis by downregulation of PDCD4 expression in human hepatocellular carcinoma cells. BMC Cancer 10: 354, 2010.

23. Cao LL, Xie JW, Lin Y, Zheng CH, Li P, Wang JB, Lin JX, Lu J, Chen QY and Huang CM: miR-183 inhibits invasion of gastric cancer by targeting Ezrin. Int J Clin Exp Pathol 7: 5582-5594, 2014.

24. Zhao H, Guo M, Zhao G, Ma Q, Ma B, Qiu X and Fan Q: miR-183 inhibits the metastasis of osteosarcoma via downregulation of the expression of Ezrin in F5M2 cells. Int J Mol Med 30: 1013-1020, 2012.

25. Xu L, Li Y, Yan D, He J and Liu D: MicroRNA-183 inhibits gastric cancer proliferation and invasion via directly targeting Bmi-1. Oncol Lett 8: 2345-2351, 2014.

26. Wang G, Mao W and Zheng S: MicroRNA-183 regulates Ezrin expression in lung cancer cells. FEBS Lett 582: 3663-3668, 2008.

27. Zhang L, Quan H, Wang S, Li X and Che X: MiR-183 promotes grow th of non-small cell lung cancer cells through FoxO1 inhibition. Tumour Biol 36: 8121-8126, 2015.

28. Wang G, Wang S and Li C: MiR-183 overexpression inhibits tumorigenesis and enhances DDP-induced cytotoxicity by targeting MTA1 in nasopharyngeal carcinoma. Tumour Biol 39: 1010428317703825, 2017. 